\title{
Multijunction Solar Cell Performance in Mars Orbiter Mission (MOM) conditions
}

\author{
$\mathrm{Uma} \mathrm{BR}^{\dagger}$, M. Sankaran, and, Suresh E. Puthanveettil \\ Power System Group, ISRO Satellite Centre (ISAC), Bangalore, India
}

${ }^{\dagger}$ Email ID: uma@isac.gov.in, Tel.no: +918025083531

\section{Introduction}

Mars Orbiter Mission (MOM) is the maiden interplanetary mission of ISRO. The mission was conceived to be a technology demonstrator and includes five payloads which are designed for the observation of the physical features of Mars and its outer atmosphere. The MOM scientific payloads are supported by mainframe systems like power, thermal, communication system, control, digital, and propulsion systems. The electrical power system of MOM comprises of a single power bus tied to a Li-ion battery and power generation is through photovoltaic solar array using state-of-art multi junction solar cells (MJSCs).

This paper discusses about the array operating requirements, array configuration, and predicted array performance versus the observed in-orbit performance at MOM orbit conditions. Also presents about the MOM array performance prediction and array observation models.

Key Words: Photovoltaic, Multi junction solar cells (MJSC), Low intensity and low temperature (LILT), solar radiation pressure (SRP).

\section{Solar Array Design Requirements}

Photovoltaic solar array (PVA) is a prime source of power for satellites not only for LEO, MEO, and GEO missions but also for various near-earth interplanetary missions and rover missions to Moon and Mars. Advances in solar cell technology in the last three to four decades, starting from the $\sim 10 \%$ efficient singlecrystal silicon solar cell to the state-of-art $\sim 28 \%$ efficient multijunction solar cells have made it possible to cater high power requirements of spacecraft and as well as it can operate in critical environment conditions. Hence, solar array has chosen to be the prime power generation source for MOM spacecraft.
MOM solar array was required to provide up to $850 \mathrm{~W}$ of spacecraft load power out of which the maximum consumption of power was of communication and an additional $375 \mathrm{~W}$ of battery charging power in all the mission phases.

Mass and hence the solar array area being at a premium for the MOM mission, use of $\sim 28 \%$ efficiency (1AM0) multijunction solar cells was the prime choice. . The dilemma of MOM solar array design was whether to design the array for the operating conditions of Martian orbit, Earth orbit or both. Our expertise suggested that the solar array be designed for Martian orbit. This would entail accounting for the off-optimal operation loss for the array during the near Earth orbit phase but optimal operation at Martian orbit. Every additional watt of power generated by the solar array, in the Martian phase means, longer periods of communication from MOM to Earth and longer mission life.

Hence, the MOM solar array was designed for the Martian phase and during the Earth bound phases, the solar array was planned to be off-set away from the sun to control the solar cell operating temperature. The mission designers were confident of ensuring this because of the following reasons:

- Battery tied configuration of the power bus

- Amount of autonomy built into MOM

- Any mission safe mode is also power safe

Such a design approach to off-set the solar array to ensure reasonable temperature of the solar cells was in well agreement with the need to minimize the Solar Radiation Pressure (SRP) torque during the cruise phase. This operation met the mission requirements throughout the Earth bound and cruise phases.

The design of the MOM mission orbit transfer strategy resulted in the spacecraft orbiting Earth several times with the low perigee altitude of near $200 \mathrm{~km}$, gaining orbital velocity with each burn of the liquid engine aboard the spacecraft. During the design phase, a worst case estimate of 88 transitions through the Van Allen 
radiation belt was envisaged. Hence, the MOM solar array design accounted for the damage due to the radiation flux through the anticipated 88 transitions through the radiation belts in addition to the predicted solar flares. In actual the MOM solar array experienced total 40 Van Allen belt crossing. The total dose of particulate radiation after each burn was estimated by considering actual orbit parameters using generic radiation models. The array performance was predicted by applying radiation remaining factors also.

With the change in solar intensity, the solar cell temperature also changes. Considering GEO spacecraft as reference, for spacecraft at large distances from the sun, the solar intensity and solar cell temperature are both low, while for spacecraft near the sun they both are high. This aggravates the difficulties of operating solar arrays both far from and near to the sun.

The MOM spacecraft during near Earth bound phase experienced $1353 \mathrm{~W} / \mathrm{m}^{2}$ and temperature of $+55^{\circ} \mathrm{C}$ similar to GEO spacecraft. In Cruise phase the spacecraft has undergone different sun intensity starting from $1400 \mathrm{~W} / \mathrm{m}^{2}$ to $700 \mathrm{~W} / \mathrm{m}^{2}$ and temperature from $+55^{\circ} \mathrm{C}$ to $-2{ }^{\circ} \mathrm{C}$. The MOM orbit around Mars features low solar irradiance $\left(493 \mathrm{~W} / \mathrm{m}^{2} \approx 0.38 \mathrm{AM} 0\right)$ and low temperature $\left(-20^{\circ} \mathrm{C}\right)$, this conditions known as Low Intensity and Low Temperature (LILT) affects the solar cell performance. In all the above orbit conditions the MOM solar array performed as per the design and prediction.

\section{MOM Solar Array Configuration}

MOM solar array is a rigid, deployable single wing array with three solar panels having total area of 7.52 $\mathrm{m}^{2}$. The panels were populated by $\sim 2,000$ space qualified multijunction solar cell assemblies of $\sim 28 \%$ BOL efficiency at RT, 1AM0. S. These solar cells are arranged into 16 switchable strings controlled by a Taper Charge Regulator (TCR) and connected to battery tied single bus. Fig.1 shows the three flight panels of the MOM mission assembly.

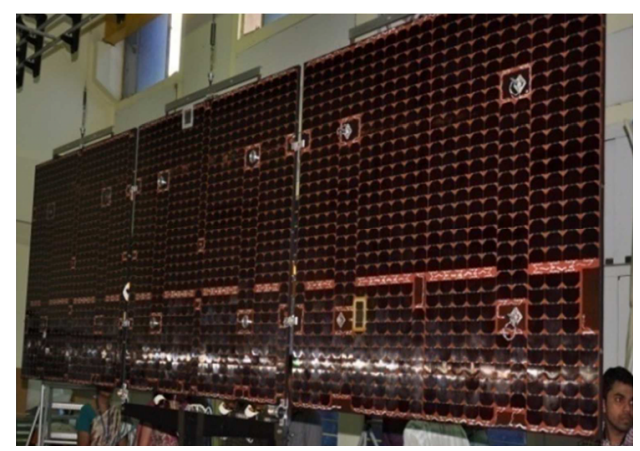

Fig1. MOM Solar Array

\section{MOM Array Performance prediction}

A solar array is Ns $\mathrm{x} \mathrm{Np}$ matrix of solar cells. Prediction of solar array power in space depends on a number of factors like type of solar cell, its characteristics, operating temperature of array, particulate radiation environment, array orientation angle, seasonal variation of sun declination angle, solar intensity and various other degradation factors. The voltage and current of the solar cell for a given solar intensity (X) and temperature (T) is defined by equations 1 to 3 .

$$
\begin{aligned}
& I_{s c}(X, T)=X \cdot I_{s c}(1, T) \\
& V_{o c}(X, T) \equiv V_{o c}(1, T)+C \cdot \ln (X) \\
& V_{L}(X, T) \equiv V_{L}(1, T)+C \cdot \ln (X)
\end{aligned}
$$

Where $\mathrm{C}$ is a constant. The solar cell parameters at different intensity and temperature throughout the mission were calculated using equations (1-3).

The generated electrical power of solar array is obtained by the following equation (4) and the solar array power prediction/ estimation model is shown in Fig 2.

$$
\begin{aligned}
& \text { Where } \\
& \mathrm{P}=\text { Power generated } \\
& \mathrm{V}=\text { Bus Voltage } \\
& \mathrm{I}=\text { Solar array Generated Current } \\
& \mathrm{i}=\text { Intensity Factor } \\
& \delta=\text { Sun declination angle } \\
& \psi=\text { Sun off set angle }
\end{aligned}
$$$$
\mathrm{P}=\mathrm{I} * \mathrm{~V} * \mathrm{i} * \operatorname{Cos}(\delta) * \operatorname{Cos}(\psi)
$$

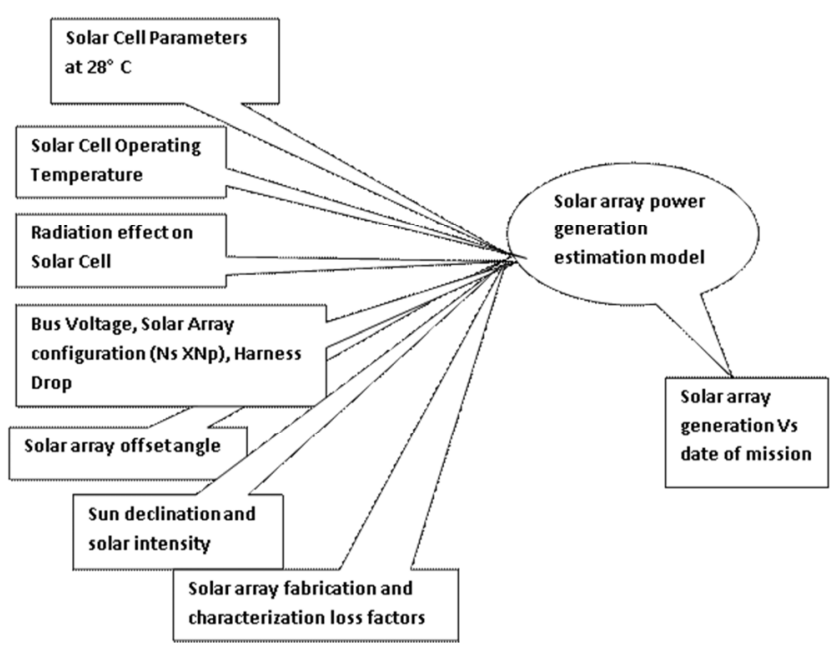

Fig2. Solar array power generation estimation model 


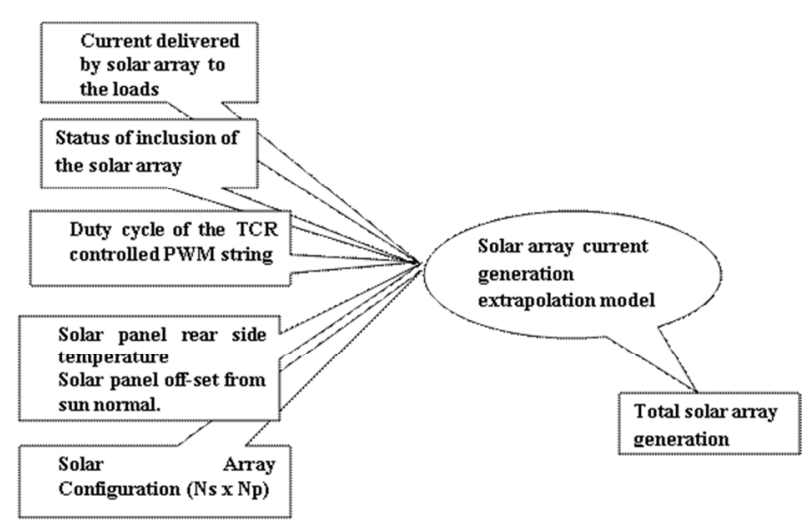

Fig3. On board solar array generation estimation model

\section{In-Orbit Performance of MOM array}

MOM spacecraft was launched on November 05, 2013 by Polar Satellite Launch Vehicle (PSLV) from SHAR, Sriharikota, India. The solar array was deployed after 45 minutes of vehicle liftoff. The solar array supported the spacecraft power requirement and battery charging requirement except during eclipse and liquid engine burn maneuvers during orbit raising operations.

The observability available on-board MOM spacecraft for performance verification of the solar array are the following:

- Current delivered by solar array to the loads/ Load Currents

- Status of inclusion/exclusion of the solar array strings

- Duty cycle of the TCR controlled PWM string

- Solar panel rear side temperature

- Solar panel off-set from sun normal.

Using these data available in telemetry, a method was established for extrapolating the total power generation capacity of MOM solar array. Such extrapolations were carried out at various points of the MOM mission. The on board array generation model is shown in fig 3 . This in-orbit telemetry derived generation estimate was compared against the theoretical predictions throughout the mission.

The solar array performance was observed for various operational conditions at different orbit phases i.e

- Different sun aspect angles of the array

- Different solar panel temperatures

- Effect of Van Allen belts transition

- Effect of LILT etc.

\section{i. Different sun aspect angles and operating temperatures}

In order to meet the design requirement of operating the solar array in Earth bound orbit phase, to control the operating temperature and also to minimize the solar radiation pressure, the solar array was operated at different offset angles from Sun and at different operating temperatures. The array performance was monitored during this phase and the array performance was matched with the prediction and is shown in Fig 4.

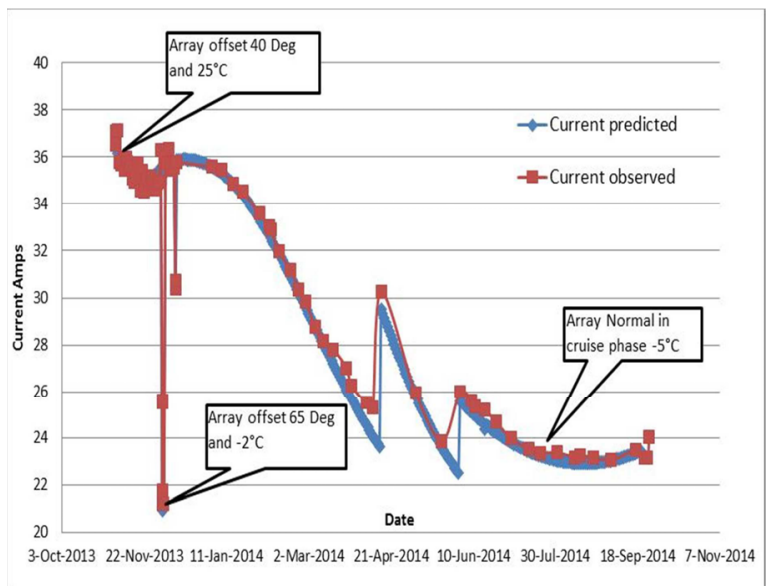

Fig4. Solar array predicted Vs observed/ telemetered performance at different offset angles and temperature in near earth orbit and cruise phases.

\section{ii. Effect of Van Allen belts transition}

Due to orbit transfer strategy to raise the MOM orbit, solar array crossed 40 Van Allen radiation belt during this period. The total dose of particulate radiation was estimated by using generic radiation models. The array performance was predicted for 32 Van Allen belt crossing by applying radiation remaining factors. The solar array performance was compared with observed data on 5th November, 2013 immediately after launch and on 21st November, 2013 after several / 32 Van Allen radiation belt crossing (after five orbit maneuvers), and datas have matched within the accuracy of $\pm 1 \%$ and are shown in fig 5 and fig 6 . 


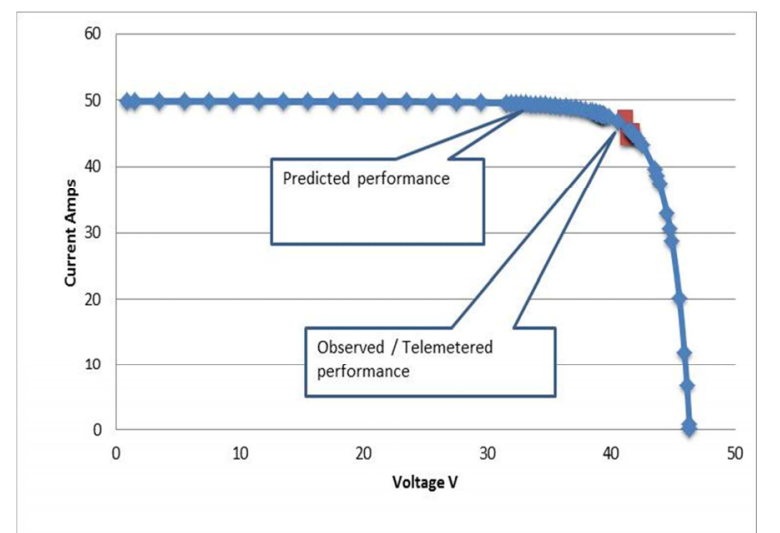

Fig5. Solar array predicted Vs observed/ telemetered performance on launch day (Nov 5, 2013)

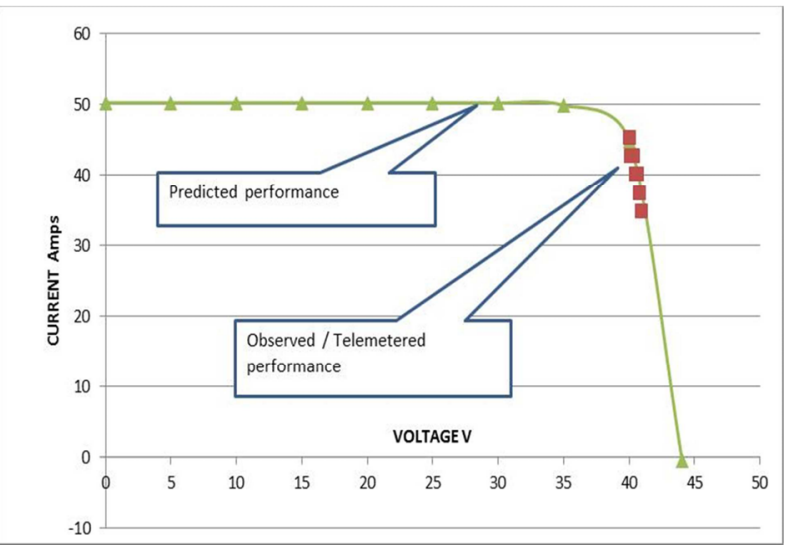

Fig6. Solar array predicted Vs observed/ telemetered performance after 32 Van Allen belt crossing

\section{iii. Effect of LILT}

Due to orbital condition of mission, MOM array has experienced low solar irradiance from $700 \mathrm{~W} / \mathrm{m}^{2}$ to $493 \mathrm{~W} / \mathrm{m}^{2} \approx 0.37 \mathrm{AM} 0$ and temperature from $-2{ }^{\circ} \mathrm{C}$ to as low as $-22^{\circ} \mathrm{C}$ conditions known as Low Intensity and Low Temperature (LILT). The observed solar solar cell performance is well matched with designed prediction within the telemetered accuracy of $2 \%$ as can be seen in fig 7 .

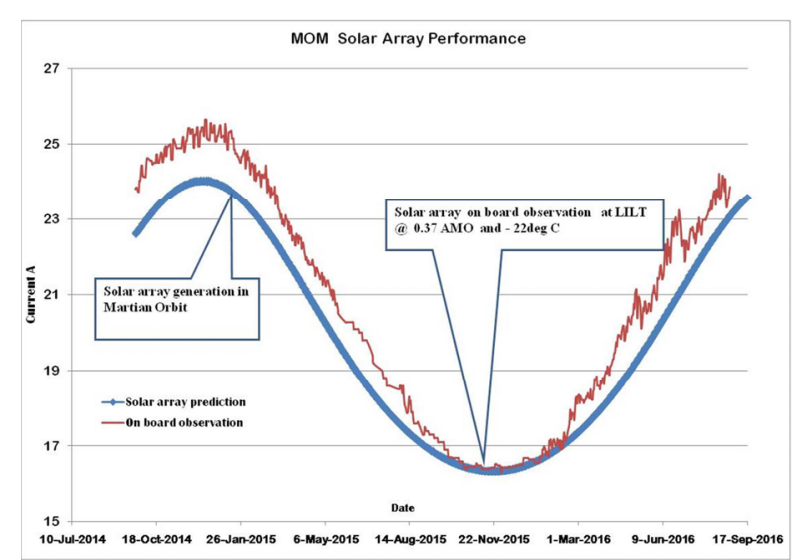

Fig7. Solar array predicted Vs observed/ performance in Martian orbit

\section{Conclusion}

A brief summary of MOM solar array design, design requirements, methodology for array performance prediction and in-orbit performance analyses at different orbit phases of MOM spacecraft were presented. The performance of the MOM solar array under all operating conditions is as per design and prediction as can be seen from the figure 4, 5, 6 and 7 throughout the mission.

\section{Acknowledgement}

The authors are grateful to Director, ISAC and Deputy Director, CPA for their guidance and support for this work. Also we wish to thank all individuals of solar panel division for their contribution in realizing the solar array for MOM.

\section{References}

1. "Solar Array Design handbook", RauschenbachH.S.,Van Nostrand Reinhold Co.,New York, USA, 1980.

2. "Solar Cell Radiation Handbook", H.Y.Tada, J.R.Carter, Jr., B.E. Anspaugh, R.G. Downing, Third Edition, JPL,NASA, California, USA, 1982 3. "Design and performance of the MER(Mars Exploration Rovers) solar arrays",Paul M.Stella, Richard C.Ewell and Julie J.Hoskin, 2005 IEEE. 4. "In Orbit performance of Ultra Triple junction solar arrays", L. Anikst,Z. Maassarani et.al. 2013 IEEE 